\title{
Effects of Ag Addition on Some Physical Properties of Granular $\mathrm{YBa}_{2} \mathrm{Cu}_{3} \mathrm{O}_{7-\delta}$ Superconductor
}

Paula de Azambuja, Pedro Rodrigues Júnior, Alcione Roberto Jurelo, ${ }^{*}$ Francisco

Carlos Serbena, Carlos Eugênio Foerster, and Rosângela Menegotto Costa Departamento de Física, Universidade Estadual de Ponta Grossa,

Av. Gen. Carlos Cavalcanti 4748, 84.030-000, Ponta Grossa, Paraná, Brazil

Gelson Biscaia de Souza

Departamento de Ciências, Universidade Estadual de Maringá, av. Colombo, 5790, Maringá, PR, CEP 87020-900, Brazil

Carlos Maurcio Lepienski

Departamento de Física, Universidade Fedral do Paraná, Centro Politécnico, C.P. 19044, Curitiba, PR, CEP 81531-990, Brazil

Adilson Luiz Chinelatto

Departamento de Engenharia de Materiais, Universidade Estadual de Ponta Grossa, Av. Gen. Carlos Cavalcanti 4748, 84030-000, Ponta Grossa, Paraná, Brazil

(Received on 21 April, 2008)

\begin{abstract}
The effects of $\mathrm{Ag}$ addition on some physical properties of $\mathrm{YBa}_{2} \mathrm{Cu}_{3} \mathrm{O}_{7-\delta}$ superconductor has been studied. The samples were produced by different routes and characterized by scanning electron microscopy, differential thermal analysis, thermogravimetric analysis, X-ray diffraction and nanoindentation. The superconducting properties were studied by $d c$ magnetization and electrical resistivity measurements. The $\mathrm{X}$-ray patterns revealed that all samples were polycrystalline and corresponded to the orthorhombic $\mathrm{YBa}_{2} \mathrm{Cu}_{3} \mathrm{O}_{7-\delta}$ phase. However, for some samples, silver appears to be present within the grains. The superconducting properties were not significantly influenced by Ag addition. Hardness and elastic modulus were also obtained by instrumented indentation. $\mathrm{Ag}$ addition was found to be effective in improving the mechanical properties, probably by filling the pores in the grain boundaries. Hardness profiles indicated values between 3.1 and $3.4 \mathrm{GPa}$ at deep tip penetration depths. The highest elastic modulus of $146 \mathrm{GPa}$ was attained for samples prepared using $\mathrm{Y}_{2} \mathrm{O}_{3}, \mathrm{BaCO}_{3}, \mathrm{CuO}$ and $\mathrm{Ag}_{2} \mathrm{O}$ as precursors, while the lowest modulus $(125 \mathrm{GPa})$ was obtained for the pure $\mathrm{YBa}_{2} \mathrm{Cu}_{3} \mathrm{O}_{7-\delta}$ sample.
\end{abstract}

Keywords: High- $T_{C}$ Superconductor; Polycrystalline; YBCO; Ag Addition

\section{INTRODUCTION}

For most applications of high-temperature superconductors (HTSC's), it is desirable good superconducting and mechanical properties. It has been reported that silver addition is effective in improving mechanical properties of the brittle superconducting oxides $\mathrm{YBa}_{2} \mathrm{Cu}_{3} \mathrm{O}_{7-\delta}$ (Y-123) [1-3]. For example, studies on textured Ag doped Y-123 samples showed that the $\mathrm{Ag}$ addition improved fracture toughness and the resistance to thermal shock [4]. At mesoscopic level, silver fills the intergranular spaces and increases plasticity and the resistance to crack formation [5].

It has been found that $\mathrm{Ag}$ enhances the critical current density and magnetic-flux trapping [6,7]. Also, silver improves the grain growth and helps to obtain a better grain orientation, lowering the electrical resistance in the normalstate $[8,9]$. In a general manner, silver addition to Y123 clearly enhances the process dominated by the weaklinks [10].

Silver can be introduced into the samples by various ways, such as by mixing with metallic $\mathrm{Ag}$, by mixing with $\mathrm{AgO} \mathrm{O}_{2}$ or by electrochemical methods $[11,12]$. Due to its excellent chemical compatibility, the silver ion can be encountered into the Y-123 grains $[7,13]$. Yet, as copper and silver

${ }^{*}$ Electronic address: arjurelo@uepg.br are from the same group of the periodic table, $\mathrm{Ag}$ atoms can substitute $\mathrm{Cu}(1)$ in the Y-123 grains and consequently many physical properties are affected by presence of microscopic granularity [14]. However, some authors $[15,16]$ also report that $C u$ sites are not substituted by $\mathrm{Ag}$ ions and the excess of $\mathrm{Ag}$ fills the pores in the bulk.

Studies devoted to Ag addition in Y-123 samples have shown its numerous advantages. The aim of this study is to produce and characterize pellets of silver doped Y-123 by different routes. The samples were characterized by scanning electron microscopy, differential thermal analysis (DTA), thermogravimetric analysis (TGA), X-ray diffraction, $d c$ magnetization and electrical resistivity measurements. Also, the hardness and elastic modulus were measured by instrumented indentation.

\section{EXPERIMENTAL DETAILS}

A series of samples were prepared by a conventional solidstate reaction using high-purity powders of $\mathrm{Y}_{2} \mathrm{O}_{3}, \mathrm{BaCO}_{3}$, $\mathrm{CuO}, \mathrm{Ag}_{2} \mathrm{O}$ and metallic Ag. We prepared four different groups of samples. Group A (labeled GA) consisted of pure Y-123 samples prepared by a solid-state reaction technique, using starting materials of $\mathrm{Y}_{2} \mathrm{O}_{3}, \mathrm{BaCO}_{3}$, and $\mathrm{CuO}$. Appropriate amounts were mixed and calcinated in air at 870,900 and $920{ }^{\circ} \mathrm{C}$ for 24 hours, and then slowly cooled through $700{ }^{0} \mathrm{C}$. Finally, samples were heated in flowing oxygen at 

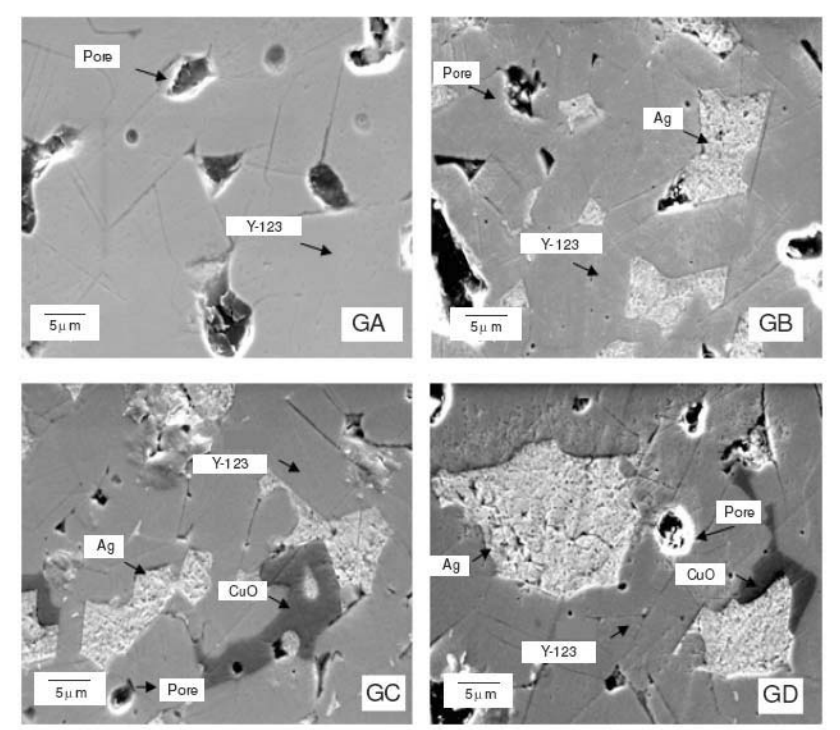

FIG. 1: SEM micrographs of a pure Y-123 (GA) and Ag-doped Y123 samples (GB, GC and GD).

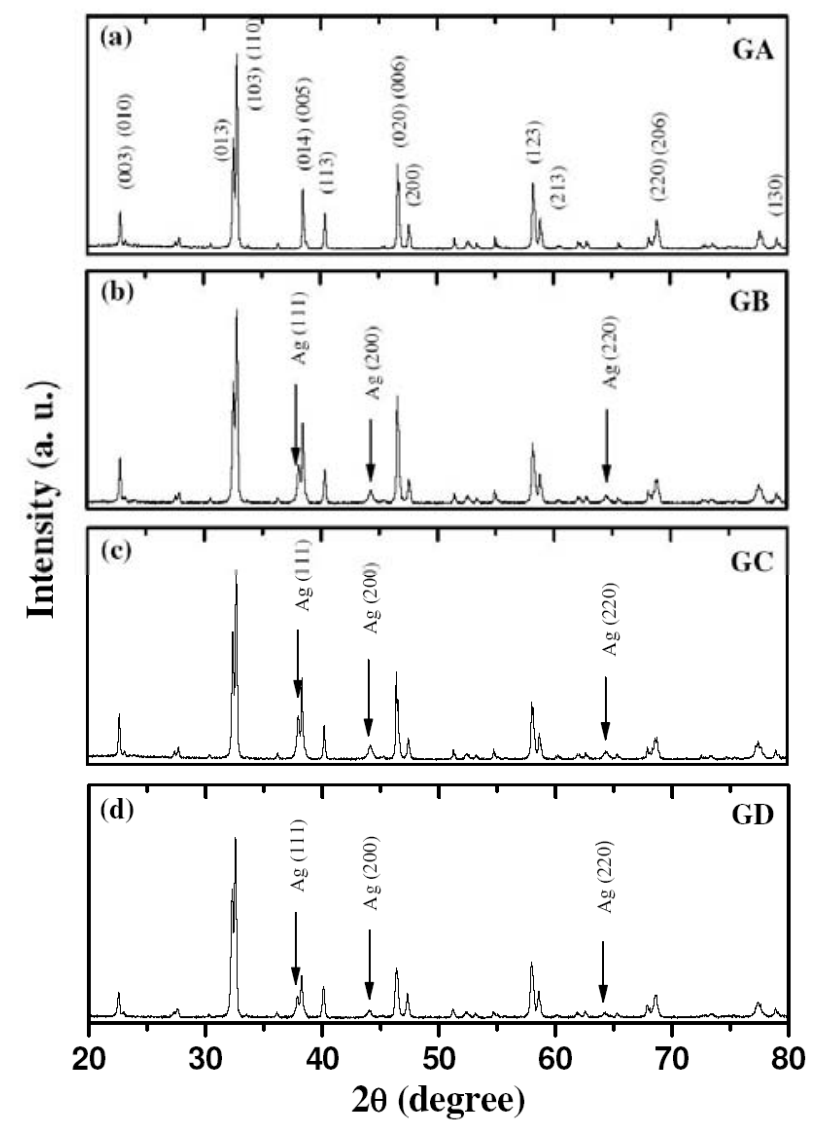

FIG. 2: Representative X-ray diffraction patterns for a pure Y-123 (GA) and for Ag-doped Y-123 samples (GB, GC and GD) prior to sintering.
$420{ }^{0} \mathrm{C}$ for 24 hours. In group $B(G B)$, a fully oxygenated 1:2:3 compound was first prepared by the usual solid-state reaction method described above. Samples of composition $\mathrm{YBa}_{2} \mathrm{Cu}_{3} \mathrm{O}_{7-\delta} / \mathrm{Ag}$, with $\mathrm{Ag}_{2} \mathrm{O} 20$ wt. \% were then prepared from $\mathrm{Ag}_{2} \mathrm{O}$ and 1:2:3 powders as described in Equation (1). The $\mathrm{Ag}_{2} \mathrm{O}$ and stoichimetric $Y-123$ powders were well mixed, pressed into pellets and sintered at $920{ }^{\circ} C$ for 24 hours. Samples were heated in flowing oxygen at $420{ }^{\circ} \mathrm{C}$ for 24 hours.

$$
\begin{aligned}
& \mathrm{YBa}_{2} \mathrm{Cu}_{3} \mathrm{O}_{7-\delta}+ \\
& +20 w t \% \mathrm{Ag}_{2} \mathrm{O} \rightarrow \mathrm{YBa}_{2} \mathrm{Cu}_{3} \mathrm{O}_{7-\delta} / \mathrm{Ag}(\mathrm{GB})
\end{aligned}
$$

Samples in group $C(G C)$ were prepared with the same chemical formula as in group A, but instead of using the prereacted 1:2:3, we used $\mathrm{Y}_{2} \mathrm{O}_{3}, \mathrm{BaCO}_{3}, \mathrm{CuO}$ and $\mathrm{Ag}_{2} \mathrm{O}$ as precursors as described in Equation (2) below.

$$
\begin{aligned}
& 1 / 2 \mathrm{Y}_{2} \mathrm{O}_{3}+2 \mathrm{BaCO}_{3}+3 \mathrm{CuO}+ \\
& 20 w t \% \mathrm{Ag}_{2} \mathrm{O} \rightarrow \mathrm{YBa}_{2} \mathrm{Cu}_{3} \mathrm{O}_{7-\delta} / \mathrm{Ag}(G C)
\end{aligned}
$$

In group $D(G D)$, samples of composition $\mathrm{YBa}_{2} \mathrm{Cu}_{3} \mathrm{O}_{7-\delta} / \mathrm{Ag}$ with pure $A g$ (metallic) $20 \mathrm{wt} \%$ were prepared from $\mathrm{Ag}$ and the prereacted 1:2:3 powders as described by Equation (3). The Ag and stoichimetric Y-123 powders were well mixed, pressed into pellets and sintered at $920{ }^{\circ} C$ for 24 hours. Samples were heated in flowing oxygen at $420{ }^{\circ} \mathrm{C}$ for 24 hours.

$$
\begin{aligned}
& \mathrm{YBa}_{2} \mathrm{Cu}_{3} \mathrm{O}_{7-\delta}+ \\
& +20 w t \% \mathrm{Ag} \rightarrow \mathrm{YBa}_{2} \mathrm{Cu}_{3} \mathrm{O}_{7-\delta} / \mathrm{Ag}(\mathrm{GD})
\end{aligned}
$$

The X-ray diffraction patterns were collected from $10^{0}$ to $100^{\circ}$ in the $2 \theta$ range with $0.02^{\circ}$ steps and $4 \mathrm{~s}$ counting time. The measurements were performed using a Shimadzu X-ray diffractometer with $C u K_{\alpha}$ radiation and the crystal structure analyses were performed using the GSAS program [17] with the EXPGUI interface [18] and the Le Bail method. Also, the identification of secondary phases was determined by micropobre energy dispersive spectroscopy (EDS). DTA and TGA measurements were carried out in a microbalance (SDT Q600 V 7.0 Build 84) under $O_{2}$ flow up to $1100{ }^{\circ} \mathrm{C}$ at a rate of $5^{0} \mathrm{C} / \mathrm{min}$. The electrical resistivity as a function of temperature was measured by means of a four-probe $A C$ technique at the frequency of $37 \mathrm{~Hz}$. The measuring current was limited to $100 \mathrm{~mA}$ for bar-shaped samples which were approximately $8 \times 3 \times 2 \mathrm{~mm}^{3}$. The temperature was determined with an accuracy of $0.01 \mathrm{~K}$ by precisely measuring the resistance of a Pt-100 sensor. The magnetic measurements were obtained by a superconducting quantum interference device MPMS-XL magnetometer from Quantum Design. Hardness and elastic modulus profiles were determined by using instrumented indentation technique following the Oliver \& Pharr method [19]. The diamond tip was of a Berkovich type and the applied load ranged from 1.6 to $400 \mathrm{mN}$. At the maximum load, a depth of $3 \mu m$ was reached. In order to measure fracture toughness, indentations with a cube-corner indenter and with load of $100 \mathrm{mN}$ were performed. Samples surface morphology and tip impressions were observed by scanning electron microscopy (SEM). 

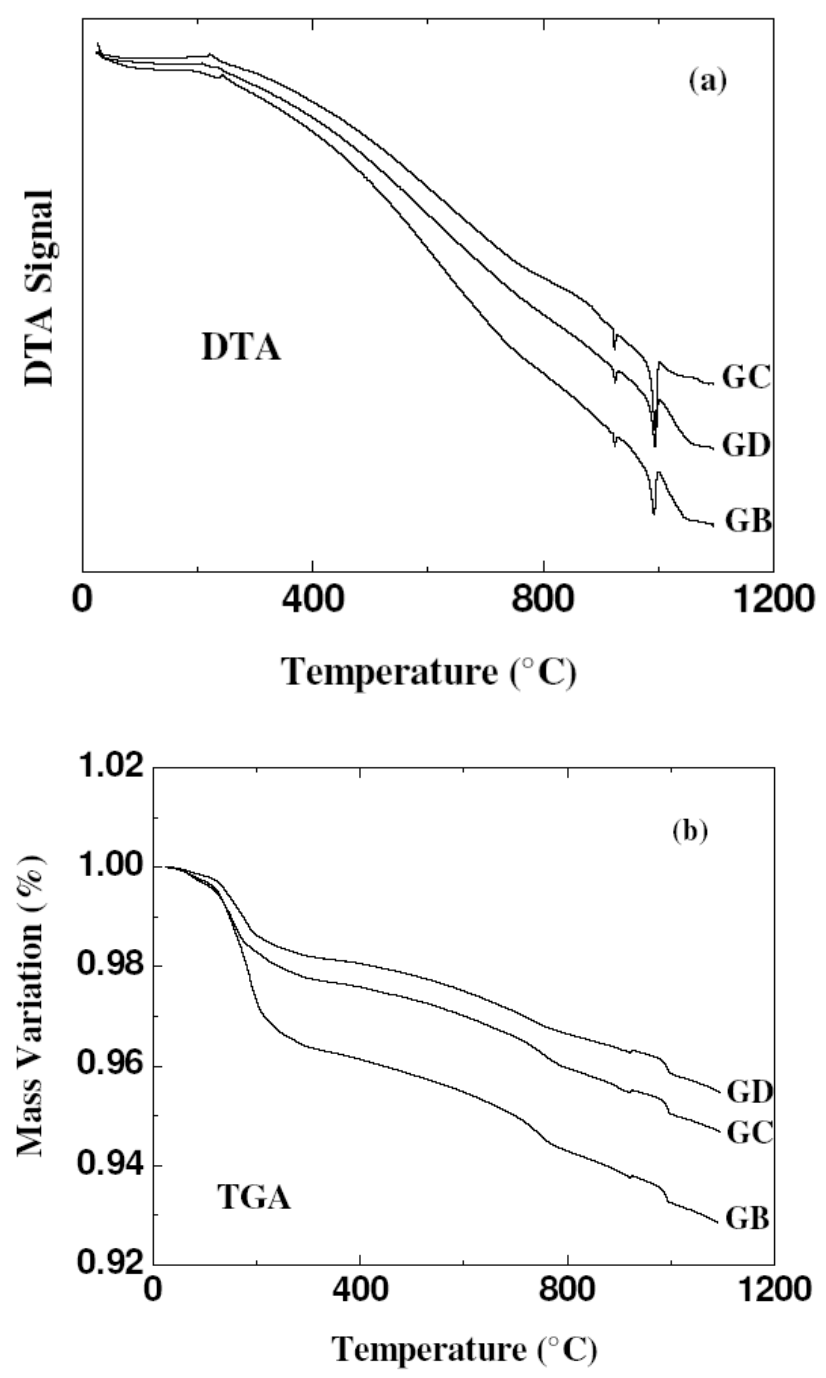

FIG. 3: (a) DTA and (b) TGA curves for Ag-doped Y-123 samples $(G B, G C$ and $G D)$.

\section{RESULTS AND DISCUSSION}

Samples polished with diamond paste finishing were observed by scanning electron microscopy. Fig. 1 shows the morphology for a pure Y-123 (sample GA) and for Ag-doped Y-123 samples $(G B, G C$ and $G D)$ polished with diamond past finishing. The granular nature and the porosity of the samples are clearly shown. For most Y-123 grains, their diameter was in range between 5 and $10 \mu \mathrm{m}$. A random distribution of metallic Ag particles between 10 and $50 \mu \mathrm{m}$ in diameter was homogeneously dispersed within the Y-123 matrix for samples $G B, G C$ and $G D$. Many defects such as pores are created during the heating and the cooling steps. Also, this kind of defect can be introduced during oxygen annealing. We can observe that the porosity for $A g$-doped samples has been significantly reduced, probably because the Ag particles fill in the pores, and with a consequent increasing in the effective density of Ag-doped samples. This result is in accordance with the porosity determined by the weight method using theoretical density (Table I). Also, for samples $G C$ and

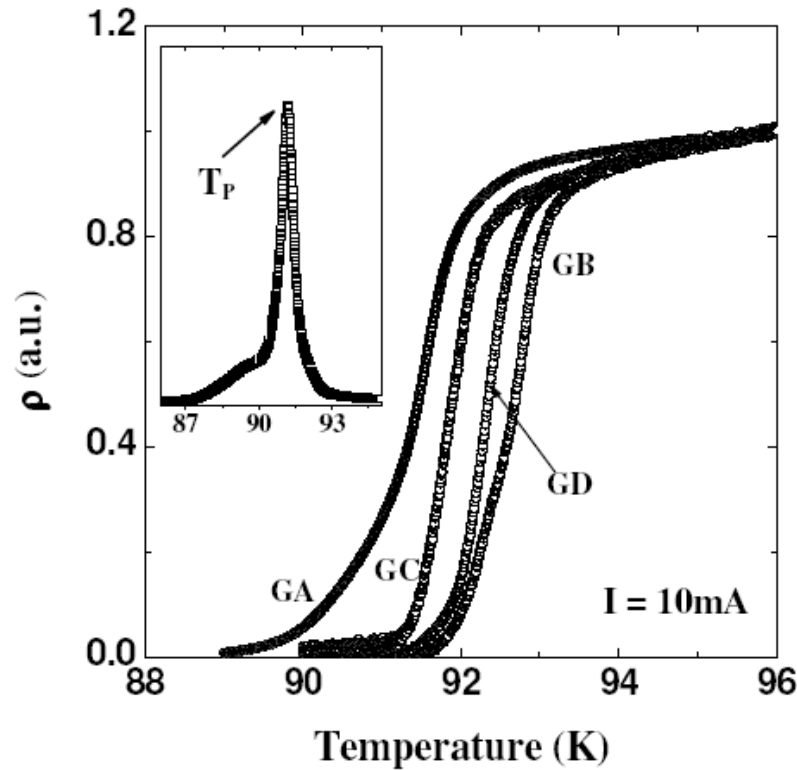

FIG. 4: Temperature dependence of the electrical resistivity of pure Y-123 $(G A)$ and Ag-doped Y-123 samples $(G B, G C$ and $G D)$. In the inset, $d \rho / d T$ versus $T$ for $G A$ sample is shown.

$G D$, we can observe the presence of $\mathrm{CuO}$, indicating probably that the substitution of $\mathrm{Cu}$ by $\mathrm{Ag}$ is important for these samples.

TABLE I: Density for pure and Ag-doped Y-123 samples.

\begin{tabular}{|c|c|}
\hline Sample & $\boldsymbol{d}\left(\mathbf{g} / \mathbf{c m}^{\mathbf{3}}\right)$ \\
\hline GA & $5.020 \pm 0.001$ \\
\hline GB & $6.007 \pm 0.009$ \\
\hline GC & $6.073 \pm 0.008$ \\
\hline GD & $5.907 \pm 0.001$ \\
\hline
\end{tabular}

The typical X-ray diffraction patterns for a pure Y123 (GA) and for Ag-doped Y-123 powders ( $G B, G C$ and $G D)$ prior to sintering are shown in Fig. 2. The X-ray patterns revealed that all powders have the orthorhombic Y-123 phase. Powders $G B, G C$ and $G D$ revealed small quantities of $A g$. The variation of lattice parameters $a, b, c$, cell volume $V$ and density $\rho$ for different samples are shown in Table II. The variation of the unit cell parameters and density can indicate that $\mathrm{Cu}$ atoms may be substituted by $\mathrm{Ag}$ atoms, which is in agreement with other studies [20,21]. Also, for $A g$-doped Y-123 samples, we could identify three silver peaks, (111), (200) and (220) [14,22], which indicates the presence of $\mathrm{Ag}$ as a separated phase of $Y-123$. However, no peaks of the $C u O$ phase were identified for powders $G C$ and $G D$, which indicates there is segregation of the $\mathrm{CuO}$ phase during sintering as seen in Fig. 1(c) and 1(d), probably by incorporation of $\mathrm{Ag}$ into the Y-123 grains.

Fig. 3 shows (a) DTA and (b) TGA data performed up to $1100{ }^{0} C$ under oxygen flow for Ag-doped Y-123 samples $(G B, G C$ and $G D)$. From DTA curves, we can observe only one endothermic peak for the melting of pure Y-123 (not shown), with the peak temperature at around $1025{ }^{\circ} \mathrm{C}$. On 


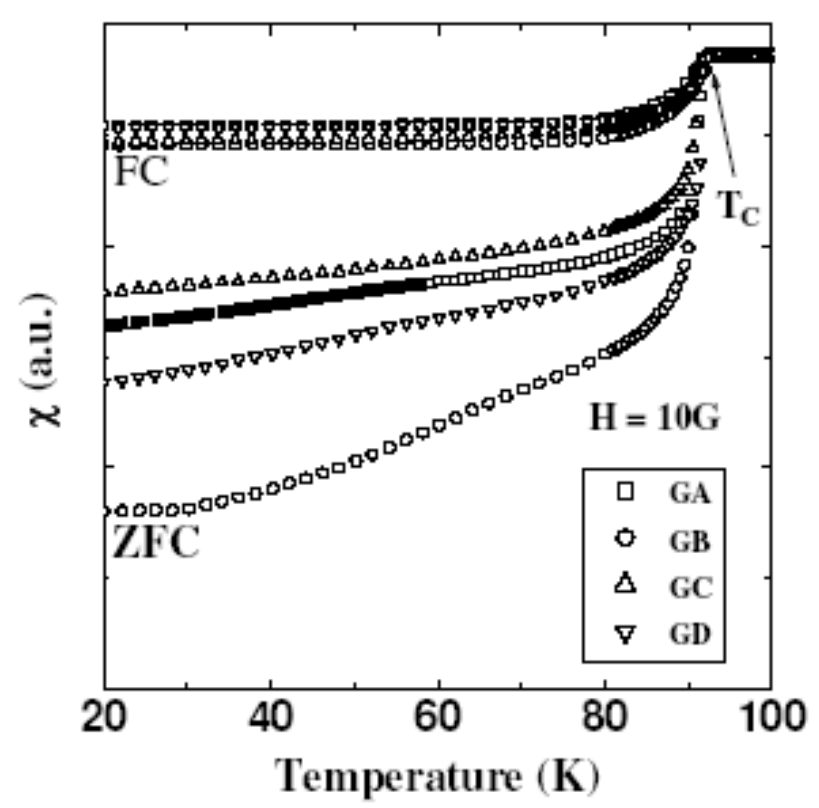

FIG. 5: FC and ZFC magnetization curves as a function of temperature for all samples measured at $H=10 \mathrm{G}$.

the other hand, with introduction of $A g$, we can observe the presence of two endothermic peaks. The first peak (lower intensity) is due to melting of bulk Ag with temperature around $950{ }^{0} \mathrm{C}$ (for all doped samples) and the peak second temperature (higher intensity)is due to Y-123 melting, that was shifted towards lower temperatures: $991{ }^{0} \mathrm{C}$ for $\mathrm{GB}, 996{ }^{0} \mathrm{C}$ for $G C$ and $992{ }^{\circ} C$ for $G D$ samples. As it was not observed others phases from DTA, the results indicated that Ag helps to lower the melting temperature of Y-123 without producing any other additional compound. Thermogravimetry of $A g$ doped Y-123 samples $(G B, G C$ and $G D)$ in oxygen has been carried out and analyzed. As can be observed from Fig. 3(b), there is a small oxygen loss around $200{ }^{0} \mathrm{C}$ for all samples, however for sample $G B$ the mass variation is higher than for the others. Also, there is a difference of mass variation between the samples. This can be attributed to the different oxygen concentration in the grain boundaries, possibly due to the use of $\mathrm{Ag}_{2} \mathrm{O}$ as a precursor.

Fig. 4 shows the temperature dependence of the electrical resistivity for pure and Ag-doped Y-123 samples near the superconductive transition. The curves are normalized to unity at $95 \mathrm{~K}$. The current was $I=10 \mathrm{~mA}$ and the values of $\rho(T)$ are within the range of the reported values for the pure and Y-123/Ag samples. Also, both pure and Ag-doped samples exhibit metallic behavior in the normal state (not shown). The transition width, $\Delta T$, defined between $5 \%$ and $95 \%$ of the transition height, is approximately $2.6 \mathrm{~K}$ for sample $G A, 1.5 \mathrm{~K}$ for $G B, 1 K$ for $G C$ and $1.2 K$ for $G D$. Yet, the zero-resistance temperature was $89.0 K$ for sample $G A, 91.5$ $K$ for $G B, 91.2 K$ for $G C$ and $91.4 K$ for $G D$. In the inset, $d \rho / d T$ is shown as a function of temperature for $G A$ sample. The derivative of the resistivity is a simple procedure for magnifying details of the transition, as for example, the maximum of $d \rho / d T$, denoted by $T_{P}$, and that corresponds approximately to the bulk critical temperature $T_{C}$ [23]. For our samples, $T_{P}$ is approximately $91.2 \mathrm{~K}$ for sample $G A, 92.7$ K for $G B, 91.8 K$ for $G C$ and $92.3 K$ for $G D$, showing that the $G B$ sample has the highest $T_{P}$. From the plot of the derivative $d \rho / d T$ versus temperature, we can observe that there is an asymmetry in $d \rho / d T$ in the temperature region below $T_{P}$ for pure Y-123 sample, indicating that the transition is a two-step process. This suggests that the pure sample must have a strong disorder at mesoscopic level $[24,25]$. On the other hand, the same behavior was not observed for Ag-doped samples, indicating much better links between superconducting grains.

Fig. 5 exemplifies typical magnetization results as a function of temperature with $H=10 \mathrm{G}$ for pure Y-123 $(G A)$ and $A g$-doped Y-123 samples $(G B, G C$ and $G D)$. The measurements were performed according to field-cooling (FC) and zero-field-cooling (ZFC) prescriptions. Meissner effect or field exclusion effect can be observed in the FC process if the sample quality is very good or defect-free. We can observe from Fig. 5 that the Meissner flux expulsion is approximately the same for all samples. Also, it is noticeable that diamagnetic signal is very small in the FC process when compared with the ZFC. It is interesting to note that $T_{C}$ is practically unaltered, $\sim 92.2-93 \mathrm{~K}$, for all samples, within our experimental resolution. Yet, the magnetic irreversibility temperature $T_{i r r}$ (the temperature corresponding to the point where $\chi_{F C}-\chi_{Z F C}$ departs from zero) also did not change significantly between the Ag-doped samples, and also as when compared with the pure compound.

Typical SEM images (Fig. 1) showed the presence of superconductors grains (Y-123), pores and microcracks. For the Ag doped samples, Ag metallic grains are present in great quantity as an embedded phase. This mixture of Y-123 phase, Ag grains and pores makes difficult to precise evaluate hardness and elastic modulus of each phase because the material is a composite. To compensate this structural inherent complexity and heterogeneity, a large ensemble of indentations was performed in order to obtain reliable hardness and elastic modulus profiles. Typical load-unloading curves for the undoped sample and Ag doped samples are shown in Fig. 6. The tip incursions observed at deeper penetrations can be attributed to pores and/or presence of Ag grains. In case of pores, the tip can lose sustentation and in case of $\mathrm{Ag}$ precipitates, a lower hardness is measured in comparison to the ceramic structure. These situations cannot be prevented during testing because both pores and Ag grains can be situated under the near surface region and are not identified when defining where to indent. Moreover, at the highest applied loads, it cannot be excluded that chipping occurs as a result of lateral crack detachment during the indentation and this also means tip sustentation lost.

Fig. 7(a) shows the hardness profiles obtained from the load-unloading curves of different samples. The hardness of the undoped sample is around $3.5 \mathrm{GPa}$ at the deepest region. The near surface region has a slightly lower hardness due to the presence of roughness. Different Ag doping processes do not modify significantly the hardness profiles in respect to the undoped condition (Table III). However, at the near surface region (up to $1000 \mathrm{~nm}$ in depth), sample $G B$ shows a slightly higher hardness in respect to the others samples, including the undoped sample. 
TABLE II: Lattice parameters $a, b, c$, unit cell volume $V$ and density $\rho$ for pure and Ag-doped Y-123 samples.

\begin{tabular}{|c|c|c|c|c|c|}
\hline Sample & $a(\AA)$ & $b(\AA)$ & $c(\AA)$ & $V\left(\mathbf{A}^{\mathbf{3}}\right)$ & $\boldsymbol{\rho}\left(\mathbf{g} / \mathbf{c m}^{\mathbf{3}}\right)$ \\
\hline $\boldsymbol{G A}$ & $3.81795 \pm$ & $3.88414 \pm$ & $11.6782 \pm$ & $173.181 \pm$ & $6.388 \pm$ \\
& 0.00008 & 0.00009 & 0.0002 & 0.009 & 0.001 \\
\hline $\boldsymbol{G B}$ & $3.8167 \pm$ & $3.8832 \pm$ & $11.6802 \pm$ & $173.11 \pm$ & $6.390 \pm$ \\
& 0.0001 & 0.0002 & 0.0003 & 0.01 & 0.001 \\
\hline $\boldsymbol{G C}$ & $3.81946 \pm$ & $3.88617 \pm$ & $11.6971 \pm$ & $173.620 \pm$ & $6.372 \pm$ \\
& 0.00008 & 0.00009 & 0.0001 & 0.006 & 0.001 \\
\hline $\boldsymbol{G D}$ & $3.82020 \pm$ & $3.8845 \pm$ & $11.6889 \pm$ & $173.457 \pm$ & $6.378 \pm$ \\
& 0.00009 & 0.0001 & 0.0003 & 0.007 & 0.001 \\
\hline
\end{tabular}

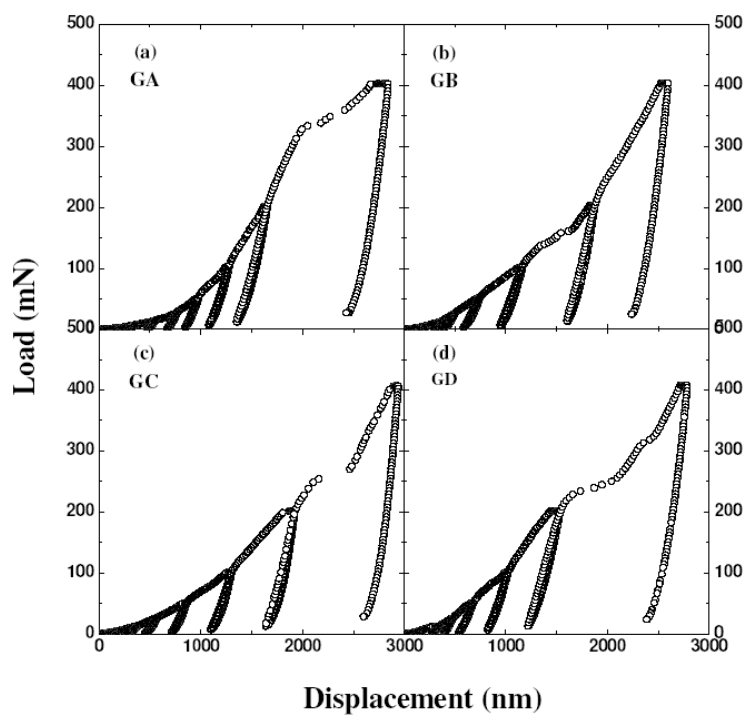

FIG. 6: Typical loading-unloading curves for pure and different Agdoped Y-123 pellets at room temperature.

All of the hardness profiles have a great dispersion in its values that can be attributed to the fact that the samples are composites. The measured hardness in these cases corresponds to an averaged behavior between the superconductor ceramic, the metallic Ag grains and the presence of defects. Taking into account this composite character of ours samples, the present hardness profiles are in agreement with reports in the literature for Y-123 based structures where hardness varies from 4 to $12 \mathrm{GPa}$ [26-32]. Consequently, the broad range of the reported values can then be attributed to the different sample processing conditions and also to the employed measurement technique, which in almost of all reported situations corresponds to the conventional Vickers method. The elastic modulus profiles for the undoped and Ag doped samples are shown in Fig. 7(b). The undoped sample has a value around $120 \mathrm{GPa}$ from near surface to deeper regions (Table III). The Ag presence increases slightly the value from 140 to $150 \mathrm{GPa}$, but as also observed in the hardness profiles, the error bars are very large for all of the studied samples. The measured values of the elastic modulus in the present situation agree with the reported data [26-32] and the same interpretation of composite behavior can be used to interpret these results.
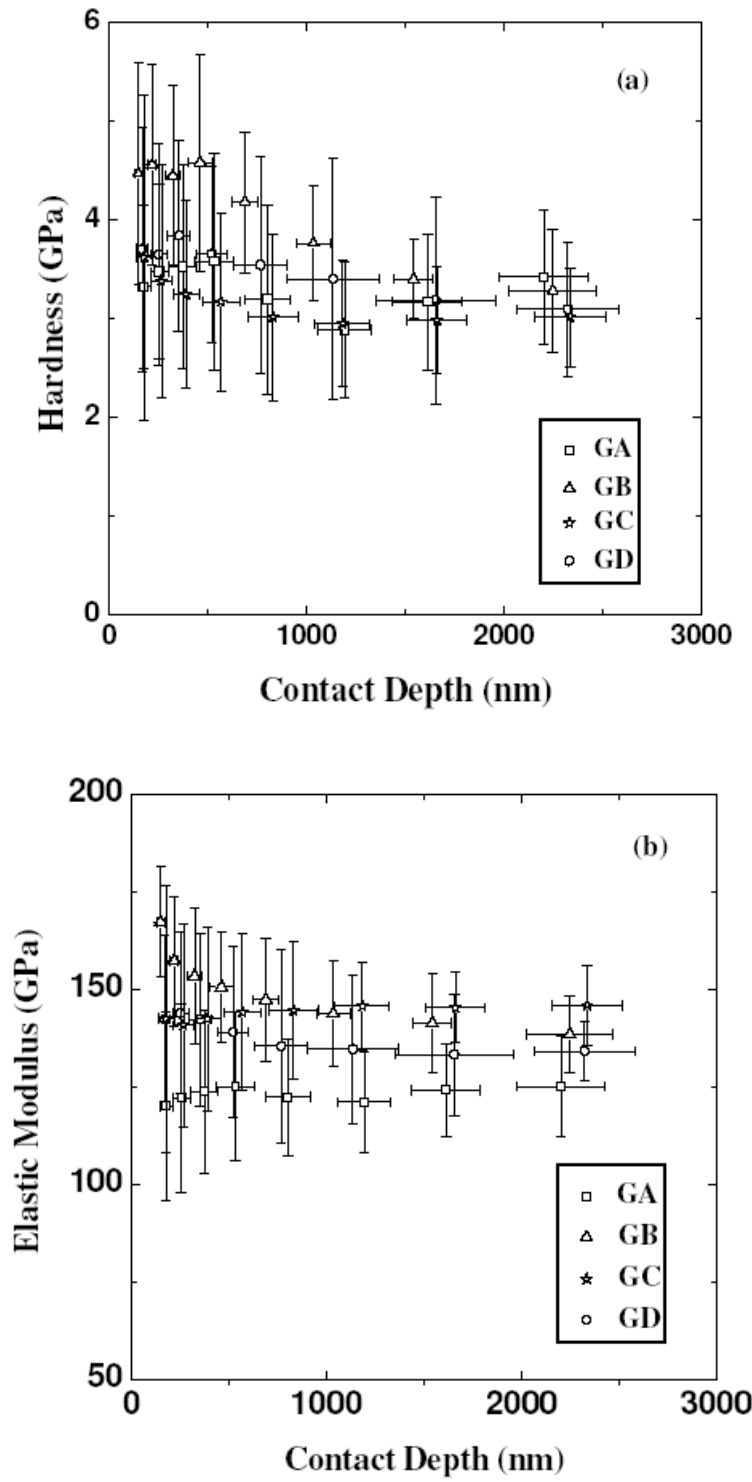

FIG. 7: (a) Hardness and (b) elastic modulus as a function of contact depth for different Y-123 samples.

Fig. 8 shows a typical cube-corner indenter impression in the sample $G C$ surface. Cracks are nucleated around the tip impression and no preferential cracking direction is ob- 


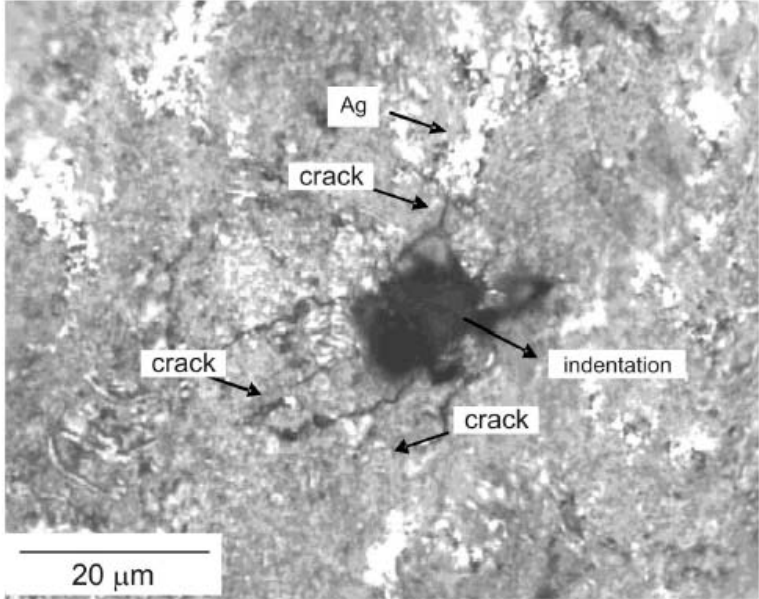

FIG. 8: Optical micrograph of a cube-corner indentation in sample GC.

TABLE III: Hardness and elastic modulus at the deepest region for pure and Ag-doped Y-123 samples measured using a Berkovich indenter.

\begin{tabular}{|c|c|c|}
\hline Sample & $\boldsymbol{H}(\mathbf{G P a})$ & $\boldsymbol{E}$ (GPa) \\
\hline GA & $3.4 \pm 0.7$ & $125 \pm 12$ \\
\hline GB & $3.3 \pm 0.6$ & $139 \pm 10$ \\
\hline GC & $3.1 \pm 0.5$ & $146 \pm 10$ \\
\hline GD & $3.1 \pm 0.7$ & $135 \pm 8$ \\
\hline
\end{tabular}

served. This behavior is in agreement with the polycrys- talline and composite nature of the samples. Radial and secondary cracks can be indentified in the figure, but due to the presence of pores and metallic Ag precipitates, the radial crack length and directions are not well defined and are very difficult to estimate. Therefore, it was not possible to measure the fracture toughness in these samples.

\section{CONCLUSION}

In the present study, the effects of the addition of $\mathrm{Ag}$ to the $\mathrm{YBa}_{2} \mathrm{Cu}_{3} \mathrm{O}_{7-\delta}$ system were investigated. Silver was added to the $\mathrm{YBa}_{2} \mathrm{Cu}_{3} \mathrm{O}_{7-\delta}$ system through the mixture with metallic $\mathrm{Ag}$ and with $\mathrm{Ag}_{2} \mathrm{O}$. Samples were produced by different routes and characterized by scanning electron microscopy, differential thermal analysis, thermogravimetric analysis and $\mathrm{X}$-ray diffraction, whereas the superconducting properties by $d c$ magnetization and electrical resistivity measurements. Also, hardness and elastic modulus were measured by instrumented indentation. It was found that $\mathrm{Cu}$ sites are substituted by $\mathrm{Ag}$ ions, as well as that metallic $\mathrm{Ag}$ also fills the pores in the grain boundaries. The results obtained are in agreement with those reported in the literature and demonstrated that the addition of silver to the $\mathrm{YBa}_{2} \mathrm{Cu}_{3} \mathrm{O}_{7-\delta}$ system is effective in improving the mechanical properties without degradation of the superconducting properties.

\section{Acknowledgements}

This work was partially financed by the CNPq Brazilian Agency under contract $n^{0} 474077 / 2007-1$.
[1] N. Sakai, A. Mase, H. Ikuta, S-J Seo, U. Mizutani, and M. Murakami, Supercond. Sci. Technol. 13, 770 (2000).

[2] C. Leblond-Harnois, R. Caillard, I. Monot-Laffez, G. Desgardin, and B. Raveau, Physica C 341, 2439 (2000).

[3] G. Plesch, I. Van Driessche, A. Cigan, J. Manka, S. Hoste, and J. Kliment, Measurement Science Review 1, 33 (2001).

[4] P. Diko, G. Fuchs, and G. Krabbes, Physica C 363, 60 (2001).

[5] J. Joo, J. G. Kim, and W. Nah, Supercond. Sci. Technol. 11, 645 (1998).

[6] O. Gorur, C. Terzoglu, A. Varilci, and M. Altunbas, Supercond. Sci. Technol. 18, 1233 (2005).

[7] E. Mendoza, T. Puig, A.E. Carrillo, J. Plain, and X. Obradors, Physica C 334, 7 (2000).

[8] J. H. Miller, S. L. Holder, J. D. Hunn, and G. N. Holder, Appl. Phys. Lett. 54, 2256 (1989).

[9] C. Harnois, Supercond. Sci. Technol. 15, 864 (2002).

[10] K. Salama, V. Selvamanickan, L. Gao, and K. Sun, Appl. Phys. Lett. 54, 2352 (1989).

[11] T. Petrisor, A. Giurgiu, and L. Ciontea, Appl. Supercond. 1, 1219 (1993).

[12] B. Ropers, F. Carmona, and S. Flandroiss, Physica C 204, 71 (1992).

[13] C. R. Taylor, and C. Greaves, Physica C 235-240, 853 (1994).

[14] Ch. Zang, A. Kulpa, and A. C. D. Chaklader, Physica C 252, 67 (1995).

[15] H. Salamati, Ali A Babaci-Brojeny, and Medhi Safa, Supercond. Sci. Technol. 14, 816 (2001).
[16] Y. Nakamura, K. Tachiban, S. Kato, T. Ban, S. I. Yoo, and H. Fujimoto, Physica C 294, 302 (1998).

[17] A.C. Larson, and R.B. Von Dreele, GSAS, Los Alamos National Laboratory (2004) Report LAUR 86-748.

[18] B. H. Toby, J. Appl. Cryst. 34, 210 (2001).

[19] W. C. Oliver, and G. M. Pharr, J. of Mat. Res. 7, 156 (1992).

[20] A. K. Gangopadhyay, and T. O. Mason, Physica C 178, 64 (1991).

[21] J. Joo, J. P. Singh, R. B. Poeppel, A. K. Gangopaddhyay, and T. O. Mason, J. Appl. Phys. 71, 2351 (1992).

[22] S. Pinol, M. Najib, T. Puig, X. Obradors, H. Xuriguera, and M. Segarra, Physica C 372-376, 738 (2002).

[23] A. R. Jurelo, I. Abrego Castillo, J. Roa-Rojas, L. M. Ferreira, L. Ghivelder, P. Pureur, and P. Rodrigues Jr., Physica C 311, 133 (1999).

[24] A. R. Jurelo, J. V. Kunzler, J. Schaf, P. Pureur, and J. Rosenblatt, Phys. Rev. B 56, 14815 (1997).

[25] F. M. Barros, F. W. Fabris, P. Pureur, J. Schaf, V. N. Vieira, A R. Jurelo, and M. P. Canto, Phys. Rev. B 73, 94516 (2006).

[26] R. R. Reddy, M. Murakami, S. Tanakam, and P. V. Reddy, Physica C 257, 137 (1996).

[27] T. Miyamato, K. Nagashima, N. Sakai, and M. Murakami, Supercond. Sci. Technol. 13, 816 (2000).

[28] Y. Yoshino, A. Iwabuchi, K. Noto, and M. Murakami, Physica C 357-360, 796 (2001).

[29] S. Sakai, S. J. Seo, K. Inoue, T. Myiamoto, and M. Murakami, Physica C 335, 107 (2000). 
[30] F. Tancret, I. Monot, and F. Osterstock, Mater. Sci. and Eng. A 298, 268 (2001).

[31] M. Matsui, N. Sakai, and M. Murakami, Supercond. Sci. Technol. 15, 1092 (2002).
[32] C. E. Foerster, E. Lima, P. Rodrigues Jr., F. C. Serbena, C. M. Lepienski, M. P. Canto, A. R. Jurelo, and X. Obradors, Braz. J. of Phys. 38, 341 (2008). 